\title{
Multivalent functions based on a linear integral operator
}

\author{
Maslina Darus and Rabha W. Ibrahim
}




\title{
MULTIVALENT FUNCTIONS BASED ON A LINEAR INTEGRAL OPERATOR
}

\author{
MASLINA DARUS AND RABHA W. IBRAHIM
}

Received 15 March, 2009

Abstract. By using a linear integral operator, a subclass of multivalent functions is introduced. Some important properties of this class such as coefficient bounds are found. Moreover, applications are also introduced.

2000 Mathematics Subject Classification: 30C45

Keywords: linear integral operator, multivalent functions, Jack's lemma

\section{INTRODUCTION}

Let $T(p)$ denote the class of functions $f(z)$ of the form

$$
f(z)=z^{p}+\sum_{n=p+1}^{\infty} a_{n} z^{n}, \quad(p \in \mathbb{N}, z \in U),
$$

which are analytic and $p$-valent (multivalent) in the open unit disk $U=\{z: z \in$ $\mathbb{C}$ and $|z|<1\}$. Given two functions $f, g \in T(p), f(z)=z^{p}+\sum_{n=p+1}^{\infty} a_{n} z^{n}$ and $g(z)=z^{p}+\sum_{n=p+1}^{\infty} b_{n} z^{n}$ their convolution or Hadamard product $f(z) * g(z)$ is defined by

$$
f(z) * g(z)=z^{p}+\sum_{n=p+1}^{\infty} a_{n} b_{n} z^{n}, \quad(z \in U) .
$$

A function $f \in T(p)$ is said to be $p$-valent starlike of order $\mu, 0 \leq \mu<p$ if

$$
\Re\left\{\frac{z f^{\prime}(z)}{f(z)}\right\}>\mu, \quad(z \in U),
$$

this class denoted by $S_{p}^{*}(\mu)$. A function $f \in T(p)$ is said to be $p$-valent convex of order $\mu, 0 \leq \mu<p$ if

$$
\Re\left\{1+\frac{z f^{\prime \prime}(z)}{f^{\prime}(z)}\right\}>\mu, \quad(z \in U),
$$

Research supported in part by UKM-ST-06-FRGS0107-2009, MOHE, Malaysia. 
this class denoted by $C_{p}(\mu)$.

For a function $f \in T(p)$ given by (1.1), we define the linear integral operator $I_{p, \alpha, \beta} f(z)$ by

$$
\begin{aligned}
I_{p, \alpha, \beta} f(z) & =(\alpha+p \beta+1) \int_{0}^{1} u^{\alpha} f\left(u^{\beta} z\right) d u \\
& =z^{p}+\sum_{n=p+1}^{\infty}\left[\frac{\alpha+p \beta+1}{\alpha+n \beta+1}\right] a_{n} z^{n}, \quad z \in U,
\end{aligned}
$$

$(\alpha, \beta \in \mathbb{R}, p \in \mathbb{N})$. Clearly, (1.2) yields

$$
f \in T(p) \Rightarrow I_{p, \alpha, \beta} \in T(p) .
$$

Thus, by applying the operator $I_{p, \alpha, \beta}$ successively, we can obtain

$$
\begin{aligned}
I_{p, \alpha, \beta}^{k} f(z) & = \begin{cases}I_{p, \alpha, \beta}\left(I_{p, \alpha, \beta}^{k-1} f(z)\right) & \text { for } k \in \mathbb{N}, \\
f(z) & \text { for } k=0,\end{cases} \\
& =z^{p}+\sum_{n=p+1}^{\infty}\left[\frac{\alpha+p \beta+1}{\alpha+n \beta+1}\right]^{k} a_{n} z^{n}, \quad(z \in U) .
\end{aligned}
$$

Note that when $p=1, \alpha=-1$ and $\beta=1$, operator (1.3) poses an integral operator defined by Sălăgean (see [9]). Further operators of type (1.2) involving different type of integral operators were introduced by many authors (cf. [1,3-5, 8]).

Let $S_{p}^{*}(\alpha, \beta, \mu)$ denote the class of all functions $f \in T(p)$ satisfying the condition

$$
\Re\left\{\frac{z\left[I_{p, \alpha, \beta}^{k} f(z)\right]^{\prime}}{I_{p, \alpha, \beta}^{k} f(z)}\right\}>\mu, \quad(0 \leq \mu<p, z \in U)
$$

and let $C_{p}(\alpha, \beta, \mu)$ be the class of all functions $f \in T(p)$ such that

$$
\Re\left\{\frac{z\left[I_{p, \alpha, \beta}^{k} f(z)\right]^{\prime \prime}}{\left[I_{p, \alpha, \beta}^{k} f(z)\right]^{\prime}}\right\}>\mu, \quad(0 \leq \mu<p, z \in U) .
$$

In the present paper, using Jack's lemma, the authors investigate the differential inequality

$$
\left|\frac{I_{p, \alpha, \beta}^{k} f(z)}{z^{p}}+\frac{z\left[I_{p, \alpha, \beta}^{k} f(z)\right]^{\prime}}{p I_{p, \alpha, \beta}^{k} f(z)}-2\right|<\mu, \quad(0 \leq \mu<p, z \in U) .
$$

Let $S_{p}^{* *}(\alpha, \beta, \mu)$ denote the class of analytic functions $f \in T(p)$ satisfying the inequality (1.4).

To establish our results, we need the following lemma given by Jack [2] (see also [7]). 
Lemma 1. Let $w(z)$ be analytic in $U$ with $w(0)=0$. If $|w(z)|$ attains its maximum value on the circle $|z|=r<1$ at a point $z_{0}$, then

$$
z_{0} w^{\prime}\left(z_{0}\right)=c w\left(z_{0}\right)
$$

where $c$ is a real number and $c \geq 1$.

\section{MAin Results}

In this section, we establish some theorems involving inequalities on $p$-valent functions. We first prove the following theorem.

Theorem 1. If $f(z) \in T(p)$ satisfies the inequality

$$
\Re\left\{1+\frac{z\left[I_{p, \alpha, \beta}^{k} f(z)\right]^{\prime \prime}}{\left[I_{p, \alpha, \beta}^{k} f(z)\right]^{\prime}}-p\right\}<\frac{2 \mu}{\mu+2}, \quad(0 \leq \mu<p, z \in U),
$$

then $f \in S_{p}^{* *}(\alpha, \beta, \mu)$.

Proof. Let the function $f(z) \in T(p)$ and satisfy the inequality (2.1). Define the function $w(z), z \in U$, such that

$$
\frac{I_{p, \alpha, \beta}^{k} f(z)}{z^{p}}=1+\frac{\mu}{2} w(z), \quad(z \in U, p \in \mathbb{N}),
$$

we have that $w(z)$ is analytic in $U$ and $w(0)=0$. Differentiating both sides of (2.2), we obtain

$$
\frac{\left[I_{p, \alpha, \beta}^{k} f(z)\right]^{\prime}}{z^{p-1}}=\frac{\mu}{2} z w^{\prime}(z)+p\left(1+\frac{\mu}{2} w(z)\right), \quad\left(z \in U, p \in \mathbb{N}, p>k, k \in \mathbb{N}_{0}\right) .
$$

Then, we have from (2.2) and (2.3) that

$$
\begin{aligned}
F(z) & :=\frac{z\left[I_{p, \alpha, \beta}^{k} f(z)\right]^{\prime}}{I_{p, \alpha, \beta}^{k} f(z)}-p \\
& =\frac{\frac{\mu}{2} z w^{\prime}(z)}{1+\frac{\mu}{2} w(z)}, \quad\left(z \in U, p \in \mathbb{N}, p>k, k \in \mathbb{N}_{0}\right) .
\end{aligned}
$$

Now, suppose that there exists a point $z_{0} \in U$ such that

$$
\max _{|z| \leq\left|z_{0}\right|}|w(z)|=\left|w\left(z_{0}\right)\right|=1 .
$$


Then, using Lemma 1 and letting $w\left(z_{0}\right)=e^{i \theta}$ such that $w\left(z_{0}\right) \neq-1$ in the equation (2.4), yields

$$
\begin{aligned}
\Re\left\{F\left(z_{0}\right)\right\} & =\Re\left\{\frac{\frac{\mu}{2} z_{0} w^{\prime}\left(z_{0}\right)}{1+\frac{\mu}{2} w\left(z_{0}\right)}\right\}=\Re\left\{\frac{\frac{\mu c}{2} w\left(z_{0}\right)}{1+\frac{\mu}{2} w\left(z_{0}\right)}\right\} \\
& =c \Re\left\{\frac{\frac{\mu}{2} e^{i \theta}}{1+\frac{\mu}{2} e^{i \theta}}\right\}=c \frac{\mu}{2+\mu} \geq \frac{\mu}{2+\mu} .
\end{aligned}
$$

On the other hand define the function $\bar{w}(z), z \in U$, such that

$$
\frac{z\left[I_{p, \alpha, \beta}^{k} f(z)\right]^{\prime}}{p I_{p, \alpha, \beta}^{k} f(z)}=1+\frac{\mu}{2} \bar{w}(z), \quad(z \in U, p \in \mathbb{N}),
$$

we find that $\bar{w}(z)$ is analytic in $U$ and $\bar{w}(0)=0$. Differentiating both sides of (2.6), yields

$$
\begin{aligned}
& \frac{z}{p}\left\{\frac{z\left[I_{p, \alpha, \beta}^{k} f(z)\right]^{\prime \prime}}{I_{p, \alpha, \beta}^{k} f(z)}-z\left(\frac{\left[I_{p, \alpha, \beta}^{k} f(z)\right]^{\prime}}{I_{p, \alpha, \beta}^{k} f(z)}\right)^{2}\right\} \\
& \quad=\frac{\mu}{2} z \bar{w}^{\prime}(z)-\left(1+\frac{\mu}{2} \bar{w}(z)\right), \quad\left(z \in U, p \in \mathbb{N}, p>k, k \in \mathbb{N}_{0}\right)
\end{aligned}
$$

Then, we have from (2.6) and (2.7)

$$
\begin{aligned}
\bar{F}(z) & :=1+\frac{z\left[I_{p, \alpha, \beta}^{k} f(z)\right]^{\prime \prime}}{\left[I_{p, \alpha, \beta}^{k} f(z)\right]^{\prime}}-\frac{z\left[I_{p, \alpha, \beta}^{k} f(z)\right]^{\prime}}{I_{p, \alpha, \beta}^{k} f(z)} \\
& =\frac{\frac{\mu}{2} z \bar{w}^{\prime}(z)}{1+\frac{\mu}{2} \bar{w}(z)}, \quad\left(z \in U, p \in \mathbb{N}, p>k, k \in \mathbb{N}_{0}\right) .
\end{aligned}
$$

Now, suppose that there exists a point $\zeta \in U$ such that

$$
\max _{|z| \leq|\zeta|}|\bar{w}(z)|=|\bar{w}(\zeta)|=1
$$

Again in view of Lemma 1 and letting $\bar{w}(\zeta)=e^{i \vartheta}$ such that $\bar{w}(\zeta) \neq-1$ in the equation (2.8), we receive

$$
\begin{aligned}
\Re\{\bar{F}(\zeta)\} & =\Re\left\{\frac{\frac{\mu}{2} \zeta \bar{w}^{\prime}(\zeta)}{1+\frac{\mu}{2} \bar{w}(\zeta)}\right\}=\Re\left\{\frac{\frac{\mu c}{2} \bar{w}(\zeta)}{1+\frac{\mu}{2} \bar{w}(\zeta)}\right\} \\
& =c \Re\left\{\frac{\frac{\mu}{2} e^{i \vartheta}}{1+\frac{\mu}{2} e^{i \vartheta}}\right\}=c \frac{\mu}{2+\mu} \geq \frac{\mu}{2+\mu} .
\end{aligned}
$$


Combining (2.5) and (2.9), we obtain

$$
\Re\left\{1+\frac{z\left[I_{p, \alpha, \beta}^{k} f(z)\right]^{\prime \prime}}{\left[I_{p, \alpha, \beta}^{k} f(z)\right]^{\prime}}-p\right\} \geq \frac{2 \mu}{2+\mu}, \quad(0 \leq \mu<p, z \in U),
$$

which contradicts the hypothesis (2.1). Therefore, we conclude that $|w(z)|<1$ and $|\bar{w}(z)|<1$ for all $z \in U$, and it yields the inequality

$$
\begin{aligned}
\left|\frac{I_{p, \alpha, \beta}^{k} f(z)}{z^{p}}+\frac{z\left[I_{p, \alpha, \beta}^{k} f(z)\right]^{\prime}}{p I_{p, \alpha, \beta}^{k} f(z)}-2\right| & \leq\left|\frac{\mu}{2} w(z)\right|+\left|\frac{\mu}{2} \bar{w}(z)\right| \\
& =\frac{\mu}{2}(|w(z)|+|\bar{w}(z)|) \\
& <\mu, \quad(0 \leq \mu<p, z \in U),
\end{aligned}
$$

that is, $f(z) \in S_{p}^{* *}(\alpha, \beta, \mu)$. Thus, the proof is completed.

Corollary 1. Let $f(z) \in T(p)$ and satisfy the inequality

$$
\Re\left\{1+\frac{z f^{\prime \prime}(z)}{f^{\prime}(z)}-p\right\}<\frac{2 \mu}{\mu+2}, \quad(0 \leq \mu<p, z \in U) .
$$

Then

$$
\left|\frac{f(z)}{z^{p}}+\frac{z f^{\prime}(z)}{p f(z)}-2\right|<\mu, \quad(0 \leq \mu<p, z \in U) .
$$

Proof. It is sufficient to put $k=0$.

Corollary 2. Let $f(z) \in T(p)$ and satisfy the inequality

$$
\Re\left\{\frac{z f^{\prime \prime}(z)}{f^{\prime}(z)}\right\}<\frac{2 \mu}{\mu+2}, \quad(0 \leq \mu<1, z \in U) .
$$

Then

$$
\left|\frac{f(z)}{z}+\frac{z f^{\prime}(z)}{f(z)}-2\right|<\mu, \quad(0 \leq \mu<1, z \in U) .
$$

Proof. It proceeds by setting $p=1$ and $k=0$.

As an application of Theorem 1, we have the following results which determine the bound estimates for functions $f \in T(p)$ to be in the class $S_{p}^{* *}(\alpha, \beta, \mu)$.

Theorem 2. Let $f(z) \in T(p)$ and satisfy

$$
\begin{aligned}
& \sum_{n=p+1}^{\infty} n((n-p)(\mu+2)+2 \mu)\left[\frac{\alpha+p \beta+1}{\alpha+n \beta+1}\right]^{k}\left|a_{n}\right| \\
&<2 p|2(\mu+1)-p(\mu+2)|, \quad(0 \leq \mu<p, z \in U) .
\end{aligned}
$$

Then $f \in S_{p}^{* *}(\alpha, \beta, \mu)$. 
Proof. Let the function $f(z) \in T(p)$ and satisfy the inequality (2.10). Thus we obtain

$$
\left|1+\frac{z\left[I_{p, \alpha, \beta}^{k} f(z)\right]^{\prime \prime}}{\left[I_{p, \alpha, \beta}^{k} f(z)\right]^{\prime}}-p\right| \leq \frac{2 p(p-1)+\sum_{n=p+1}^{\infty} n(n-p)\left[\frac{\alpha+p \beta+1}{\alpha+n \beta+1}\right]^{k}\left|a_{n}\right|}{p-\sum_{n=p+1}^{\infty} n\left[\frac{\alpha+p \beta+1}{\alpha+n \beta+1}\right]^{k}\left|a_{n}\right|}
$$

the last inequality is less than $\frac{\mu}{\mu+2}$ if the assertion (2.10) holds. Now by using the fact that $\Re\{z\} \leq|z|$ then in view of Theorem 1 yields $f \in S_{p}^{* *}(\alpha, \beta, \mu)$.

Theorem 3. Let

$$
f(z)=z^{p}+\sum_{n=p+1}^{\infty} a_{n} z^{n} \text { and } g(z)=z^{p}+\sum_{n=p+1}^{\infty} b_{n} z^{n}
$$

satisfy the inequality (2.10). Then the weighted mean of $f$ and $g$ defined by

$$
W_{i}(z)=\frac{1}{2}[(1-i) f(z)+(1+i) g(z)], \quad(0<i<1)
$$

is in $S_{p}^{* *}(\alpha, \beta, \mu)$.

Proof. By the hypotheses of the theorem then in view of Theorem 2, we have

$$
\begin{aligned}
\sum_{n=p+1}^{\infty} n((n-p)(\mu+2)+2 \mu) & {\left[\frac{\alpha+p \beta+1}{\alpha+n \beta+1}\right]^{k}\left|a_{n}\right| } \\
& <2 p|2(\mu+1)-p(\mu+2)|
\end{aligned}
$$

and

$$
\begin{aligned}
\sum_{n=p+1}^{\infty} n((n-p)(\mu+2)+2 \mu) & {\left[\frac{\alpha+p \beta+1}{\alpha+n \beta+1}\right]^{k}\left|b_{n}\right| } \\
& <2 p|2(\mu+1)-p(\mu+2)| .
\end{aligned}
$$

After a simple calculation we obtain

$$
W_{i}(z)=z^{p}+\sum_{n=p+1}^{\infty}\left[\frac{(1-i)}{2} a_{n}+\frac{(1+i)}{2} b_{n}\right] z^{n} .
$$

However,

$$
\begin{gathered}
\sum_{n=p+1}^{\infty} n((n-p)(\mu+2)+2 \mu)\left[\frac{\alpha+p \beta+1}{\alpha+n \beta+1}\right]^{k}\left|\frac{(1-i)}{2} a_{n}+\frac{(1+i)}{2} b_{n}\right| \\
\leq \sum_{n=p+1}^{\infty} n((n-p)(\mu+2)+2 \mu)\left[\frac{\alpha+p \beta+1}{\alpha+n \beta+1}\right]^{k} \frac{1-i}{2}\left|a_{n}\right|
\end{gathered}
$$




$$
\begin{aligned}
& +\sum_{n=p+1}^{\infty} n((n-p)(\mu+2)+2 \mu)\left[\frac{\alpha+p \beta+1}{\alpha+n \beta+1}\right]^{k} \frac{1+i}{2}\left|b_{n}\right| \\
= & \frac{1-i}{2} \sum_{n=p+1}^{\infty} n((n-p)(\mu+2)+2 \mu)\left[\frac{\alpha+p \beta+1}{\alpha+n \beta+1}\right]^{k}\left|a_{n}\right| \\
& +\frac{1+i}{2} \sum_{n=p+1}^{\infty} n((n-p)(\mu+2)+2 \mu)\left[\frac{\alpha+p \beta+1}{\alpha+n \beta+1}\right]^{k}\left|b_{n}\right| \\
\leq & 2 p|2(\mu+1)-p(\mu+2)| \frac{1-i}{2}+2 p|2(\mu+1)-p(\mu+2)| \frac{1+i}{2} \\
= & 2 p|2(\mu+1)-p(\mu+2)| .
\end{aligned}
$$

Consequently, by Theorem $2, W_{i}(z) \in S_{p}^{* *}(\alpha, \beta, \mu)$.

Theorem 4. Let

$$
f_{j}(z)=z^{p}+\sum_{n=p+1}^{\infty} a_{n} z^{n}, \quad j=1,2,3, \ldots, m
$$

satisfy the inequality (2.10). Then the arithmetic mean of $f_{j}(z)$ defined by

$$
F(z)=\frac{1}{m} \sum_{j=1}^{m} f_{j}(z), \quad(z \in U)
$$

is in $S_{p}^{* *}(\alpha, \beta, \mu)$.

Proof. Since $f_{j}$ satisfies (2.10) then, in view of Theorem 2, we have

$$
\begin{aligned}
\sum_{n=p+1}^{\infty} n((n-p)(\mu+2)+2 \mu) & {\left[\frac{\alpha+p \beta+1}{\alpha+n \beta+1}\right]^{k}\left|a_{n, j}\right| } \\
& <2 p|2(\mu+1)-p(\mu+2)| .
\end{aligned}
$$

A computation gives

$$
\begin{aligned}
F(z) & =\frac{1}{m} \sum_{j=1}^{m} f_{j}(z) \\
& =\frac{1}{m} \sum_{j=1}^{m}\left[z^{p}+\sum_{n=p+1}^{\infty} a_{n, j} z^{n}\right] \\
& =z^{p}+\sum_{n=p+1}^{\infty}\left[\frac{1}{m} \sum_{j=1}^{m} a_{n, j}\right] z^{n}
\end{aligned}
$$


Hence

$$
\begin{aligned}
\sum_{n=p+1}^{\infty} n & ((n-p)(\mu+2)+2 \mu)\left[\frac{\alpha+p \beta+1}{\alpha+n \beta+1}\right]^{k}\left|\frac{1}{m} \sum_{j=1}^{m} a_{n, j}\right| \\
& \leq \sum_{n=p+1}^{\infty} n((n-p)(\mu+2)+2 \mu)\left[\frac{\alpha+p \beta+1}{\alpha+n \beta+1}\right]^{k} \frac{1}{m} \sum_{j=1}^{m}\left|a_{n, j}\right| \\
& =\frac{1}{m} \sum_{j=1}^{m}\left[\sum_{n=p+1}^{\infty} n=((n-p)(\mu+2)+2 \mu)\left[\frac{\alpha+p \beta+1}{\alpha+n \beta+1}\right]^{k}\left|a_{n, j}\right|\right] \\
& <\frac{1}{m} \sum_{j=1}^{m} 2 p|2(\mu+1)-p(\mu+2)| \\
& =2 p|2(\mu+1)-p(\mu+2)| .
\end{aligned}
$$

This implies that $F(z) \in S_{p}^{* *}(\alpha, \beta, \mu)$.

Theorem 5. Let

$$
f(z)=z^{p}+\sum_{n=p+1}^{\infty} a_{n} z^{n}
$$

be in $T(p)$ such that $f(U)$ is convex. Assume that $f$ satisfies the inequality (2.10). Then, for the Cesàro operator [6,10-12] of $f$ defined by the relation

$$
\sigma_{n}(z)=\sum_{n=p+1}^{\infty} \frac{1}{n+1}\left(\sum_{i=0}^{n} a_{n, i}\right) z^{n}, \quad(p=1,2, \ldots, z \in U)
$$

with $\sigma_{0}(z)=0, \sigma_{1}(z)=z^{p}$, we have $\sigma_{n}(z) \in S_{p}^{* *}(\alpha, \beta, \mu)$.

Proof. Since $f_{j}$ satisfies (2.10) then in view of Theorem 2, we have

$$
\begin{aligned}
\sum_{n=p+1}^{\infty} n((n-p)(\mu+2)+2 \mu) & {\left[\frac{\alpha+p \beta+1}{\alpha+n \beta+1}\right]^{k}\left|a_{n, j}\right| } \\
& <2 p|2(\mu+1)-p(\mu+2)| .
\end{aligned}
$$

For all $n \in \mathbb{N}_{0}$ we have

$$
\sigma_{n}(z)=0+z^{p}+\sum_{n=p+1}^{\infty} \frac{1}{n+1}\left(\sum_{i=0}^{n} a_{n, i}\right) z^{n}, \quad(z \in U) .
$$

Hence

$$
\sum_{n=p+1}^{\infty} n((n-p)(\mu+2)+2 \mu)\left[\frac{\alpha+p \beta+1}{\alpha+n \beta+1}\right]^{k}\left|\frac{1}{n+1}\left(\sum_{i=0}^{n} a_{n, i}\right)\right|
$$




$$
\begin{aligned}
& \leq \sum_{n=p+1}^{\infty} n((n-p)(\mu+2)+2 \mu)\left[\frac{\alpha+p \beta+1}{\alpha+n \beta+1}\right]^{k} \frac{1}{n+1}\left(\sum_{i=0}^{n}\left|a_{n, i}\right|\right) \\
& =\frac{1}{n+1} \sum_{i=0}^{n}\left(\sum_{n=p+1}^{\infty} n((n-p)(\mu+2)+2 \mu)\left[\frac{\alpha+p \beta+1}{\alpha+n \beta+1}\right]^{k}\left|a_{n, j}\right|\right) \\
& <\frac{1}{n+1} \sum_{i=0}^{n} 2 p|2(\mu+1)-p(\mu+2)| \\
& =2 p|2(\mu+1)-p(\mu+2)| .
\end{aligned}
$$

This implies that $F(z) \in S_{p}^{* *}(\alpha, \beta, \mu)$.

\section{ACKNOWLEDGEMENT}

The authors express their gratitude to the Malaysian Ministry of Higher Education (MOHE) for their support in our research activities.

\section{REFERENCES}

[1] S. D. Bernardi, "Convex and starlike univalent functions," Trans. Amer. Math. Soc., vol. 135, pp. 429-446, 1969.

[2] I. S. Jack, "Functions starlike and convex of order $\alpha$," J. London Math. Soc. (2), vol. 3, pp. 469474, 1971.

[3] I. B. Jung, Y. C. Kim, and H. M. Srivastava, "The Hardy space of analytic functions associated with certain one-parameter families of integral operators," J. Math. Anal. Appl., vol. 176, no. 1, pp. 138-147, 1993. [Online]. Available: http://dx.doi.org/10.1006/jmaa.1993.1204

[4] J.-1. Liu, "On a class of $p$-valent analytic functions," Chinese Quart. J. Math., vol. 15, no. 4, pp. $27-32,2000$.

[5] J.-L. Liu, "Some applications of certain integral operator," Kyungpook Math. J., vol. 43, no. 2, pp. 211-219, 2003

[6] J. Miao, "The Cesàro operator is bounded on $H^{p}$ for $0<p<1$," Proc. Amer. Math. Soc., vol. 116, no. 4, pp. 1077-1079, 1992. [Online]. Available: http://dx.doi.org/10.2307/2159491

[7] S. S. Miller and P. T. Mocanu, "Second-order differential inequalities in the complex plane," J. Math. Anal. Appl., vol. 65, no. 2, pp. 289-305, 1978. [Online]. Available: http://dx.doi.org/10.1016/0022-247X(78)90181-6

[8] S. Owa and H. M. Srivastava, "Some applications of the generalized Libera integral operator," Proc. Japan Acad. Ser. A Math. Sci., vol. 62, no. 4, pp. 125-128, 1986. [Online]. Available: http://projecteuclid.org/getRecord?id=euclid.pja/1195514368

[9] G. Ş. Sălăgean, "Subclasses of univalent functions," in Complex analysis—fifth Romanian-Finnish seminar, Part 1 (Bucharest, 1981), ser. Lecture Notes in Math. Berlin: Springer, 1983, vol. 1013, pp. 362-372. [Online]. Available: http://dx.doi.org/10.1007/BFb0066543

[10] J.-h. Shi and G.-b. Ren, "Boundedness of the Cesàro operator on mixed norm spaces," Proc. Amer. Math. Soc., vol. 126, no. 12, pp. 3553-3560, 1998. [Online]. Available: http://dx.doi.org/10.1090/S0002-9939-98-04514-6

[11] A. G. Siskakis, "Composition semigroups and the Cesàro operator on $H^{p}$," J. London Math. Soc. (2), vol. 36, no. 1, pp. 153-164, 1987. [Online]. Available: http://dx.doi.org/10.1112/jlms/s2-36. 1.153 
[12] A. G. Siskakis, "The Cesàro operator is bounded on $H^{1}$," Proc. Amer. Math. Soc., vol. 110, no. 2, pp. 461-462, 1990. [Online]. Available: http://dx.doi.org/10.2307/2048089

\section{Authors' addresses}

Maslina Darus

School of Mathematical Sciences, Faculty of Science and Technology, University Kebangsaan Malaysia, Bangi 43600, Selangor Darul Ehsan, Malaysia

E-mail address: maslina@ukm.my

\section{Rabha W. Ibrahim}

School of Mathematical Sciences, Faculty of Science and Technology, University Kebangsaan Malaysia, Bangi 43600, Selangor Darul Ehsan, Malaysia

E-mail address: E-mail: rabhaibrahimeyahoo.com 